\title{
Near infrared photography of atmospheric optical phenomena
}

\author{
Joseph Shaw, Michael Vollmer
}

Joseph A. Shaw, Michael Vollmer, "Near infrared photography of atmospheric optical phenomena," Proc. SPIE 11143, Fifteenth Conference on Education and Training in Optics and Photonics: ETOP 2019, 111431P (2 July 2019); doi: $10.1117 / 12.2523165$ 


\title{
Near infrared photography of atmospheric optical phenomena
}

\author{
Joseph A. Shaw*a, Michael Vollmer ${ }^{\mathrm{b}}$ \\ ${ }^{a}$ Montana State University, P.O. Box 173515, Bozeman, MT USA 59717-3515 \\ bUniversity of Applied Sciences Brandenburg, Magdeburgerstr. 50, 14770 Brandenburg, Germany
}

\begin{abstract}
Mirages, rainbows, halos, glories, and coronas are well-known atmospheric optics phenomena that can be used as examples when teaching the well-understood underlying optical principles of refraction, reflection, dispersion, diffraction, and scattering. These beautiful natural phenomena can generate interest when used for teaching optics because they usually are easily observable with the naked eye. The invention of Si-sensor-based digital photography, however, also offers easy investigations in spectral regions adjacent to the visible range. We report and discuss observations of natural phenomena with a modified DSLR camera operating in the near infrared spectral range above $800 \mathrm{~nm}$. This investigation may be particularly useful in photonics education, as the discussion of photon detectors in certain wavelength ranges can be combined with atmospheric optics, which always attracts interest in students.
\end{abstract}

Keywords: optics education, atmospheric optics, NIR photography

\section{INTRODUCTION}

Rainbows, halos, coronas, glories, and mirages are well known beautiful natural phenomena. ${ }^{1-3}$ These naked eye observable features are particularly suitable for teaching optics. From a physics point of view, using a camera to record images rather than the eye for observing the phenomena just means that the detector signal, based on its spectral response, is changed. Using detectors in adjacent regions to the visible (VIS) range will result in images that may differ appreciably from the visual appearance owing to the different detector spectral response and the wavelength-dependent light scattering processes in the atmosphere. Presenting example images and discussing the differences between the VIS and near infrared (NIR) features provides a different and attractive approach to teach the relevant physical processes responsible for producing the phenomena.

To illustrate this approach, we used a modified digital single-lens reflex (DSLR) camera with the IR-blocking filter removed from the front of the detector, making the camera operate in the NIR. ${ }^{4}$ Here we present observations of atmospheric optical phenomena with such a modified camera operating in the NIR region above $800 \mathrm{~nm}$ wavelengths. ${ }^{5}$ Other characteristics of NIR photography have been discussed recently., ${ }^{4,6-8}$ In this work, we refer to NIR as the wavelength range from $780 \mathrm{~nm}$ to $1100 \mathrm{~nm}$, which can be nicely covered by Si-based sensors.

\section{POTENTIAL DIFFERENCES IN NIR AND VIS OPTICAL PHENOMENA}

Any explanation of optical phenomena recorded by a camera can be divided into the various interactions of the radiation from the source on its way towards the observer (Fig. 1). Each interaction may depend on wavelength and may thus lead to differences between observations of the VIS and the NIR phenomenon. The radiation must pass through the atmosphere to reach the observed object (molecule, water droplet, ice crystal, aerosol), which scatters and/or absorbs radiation to produce the natural optical phenomenon being observed. Next, the radiation must pass through the atmosphere from object to observer before it is detected by the camera sensor.

For atmospheric optics, the natural light sources are the Sun or Moon. Here, we deal with solar radiation only. Within its spectrum there is ample NIR radiation available between wavelengths of $800 \mathrm{~nm}$ and $1100 \mathrm{~nm},{ }^{5}$ which may be used in daylight NIR photography. There is some attenuation of this NIR radiation while passing through the atmosphere; however, there is still enough NIR radiation available for recording natural scenes. ${ }^{5}$

*joseph.shaw@montana.edu; phone 1406 994-7261; fax 1406 994-5859; http://www.montana.edu/jshaw/

Fifteenth Conference on Education and Training in Optics and Photonics: ETOP 2019, edited by

Anne-Sophie Poulin-Girard, Joseph A. Shaw, Proc. of SPIE Vol. 11143, 111431P · C) 2019

SPIE, ICO, IEEE, OSA · CCC code: 0277-786X/19/\$18 · doi: 10.1117/12.2523165 


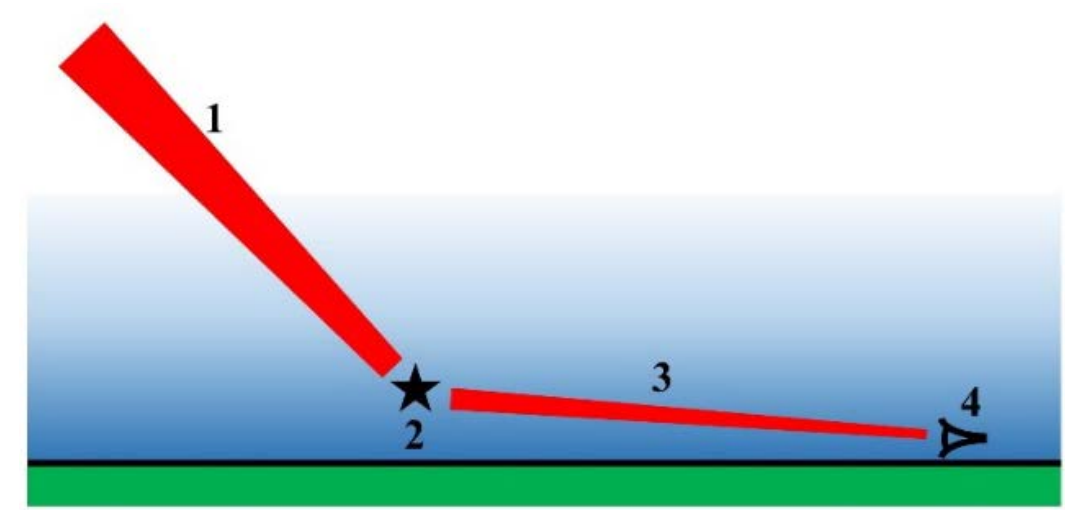

Figure 1. Scheme of how incoming radiation is modified before being detected: 1) path from Sun to object; 2) object absorption and/or scattering; 3) path from object to observer; 4) detection.

When it impinges on the objects responsible for the various phenomena (e.g. water drops for rainbows, ice crystals for halos, molecules for mirages), there will be wavelength-dependent scattering angles and attenuation resulting from the scattering process. Here, we assume that the basic physics explanations of the individual phenomena are already well known and discuss only the differences between the VIS and NIR region. For example, rainbows and halos require the light to pass through water and ice and the question arises whether NIR radiation is differently attenuated compared to visible wavelengths. For typical dimensions of water drops and ice crystals in the atmosphere, water and ice absorption differences are still small between the VIS and NIR ranges, which means that NIR observation of rainbows and halos should not be greatly altered (although they can be prohibited for longer infrared wavelengths). ${ }^{5}$

After being scattered by the object, radiation must pass through the atmosphere to the observer. Distances can vary from hundreds of meters to kilometers. As mentioned before, NIR solar radiation passing through the atmosphere along a near-vertical path is not greatly affected. In this case, however, the path is much closer to the ground, where atmospheric pressure is higher and the air can accommodate more water vapor, a well-known greenhouse gas that absorbs in the IR range. Indeed, a water molecule absorption feature between $900 \mathrm{~nm}$ and $1000 \mathrm{~nm}$ is prominent in the NIR spectrum. ${ }^{5}$ However, the spectral features are narrow and only a small part of the NIR band is affected. Therefore, if broadband NIR radiation between $800 \mathrm{~nm}$ and $1100 \mathrm{~nm}$ is detected, the overall effect may just be a small decrease in image brightness.

The ultimate detection of radiation is then done by the silicon CCD or CMOS sensor in a camera. The same sensor can record either VIS or NIR images through the use of different spectral filters in front of the detector. A normal camera usually blocks any NIR radiation and transmits VIS light, whereas the NIR cameras block all the VIS radiation and transmit NIR radiation. The color-producing Bayer filters for RGB are usually still there, as they generally transmit NIR radiation. ${ }^{4,5}$ However, they result in slightly differing spectral sensitivities in the NIR, with just a little bit more signal from the red pixels relative to the green and blue pixels; i.e., NIR images generally will have a monochrome appearance with a slight reddish tint. Usually, the resulting images are converted to gray scale.

\section{NEAR INFRARED OBSERVATIONS OF ATMOSPHERIC OPTICAL PHENOMENA}

In the visible spectral range, phenomena like rainbows or refraction halos are quite easy to see because of their pronounced color spread as a function of scattering angle. For monochrome detection in the NIR, however, this information is missing, so these phenomena must be detected via their brightness variation with respect to the background. A homogeneous dark background is desirable, but in practice one may have to deal with inhomogeneous backgrounds, such as a sky with scattered cloud fragments of variable optical depth or a landscape with varying vegetation. The latter can also illustrate another NIR-specific problem: dark VIS background may not mean dark NIR background. For example, a dark green vegetation background is helpful for recording a VIS rainbow image; however, the green vegetation appears extremely bright in the NIR, so the rainbow signal-to-background ratio is much lower in the NIR than in the VIS, easily making the NIR rainbow not detectable. In the following we present examples for four different atmospheric optical phenomena. 


\subsection{Rainbows}

The first and most well-known example of an NIR atmospheric optical phenomenon is the near infrared rainbow, which was first recorded as a still image on film. ${ }^{9}$ The NIR primary rainbow angle at $900 \mathrm{~nm}$ is around $42.7^{\circ}$ and thus within $1^{\circ}$ of the VIS rainbow angles (e.g. $42.0^{\circ}$ at $600 \mathrm{~nm}$ ). The corresponding secondary rainbow changes from $51.0^{\circ}$ to $49.7^{\circ}$.

NIR rainbows are more difficult to record than VIS rainbows because rainbows are rare and transient phenomena, so a NIR camera must therefore always be available and easy to set up using a tripod (exposure times in the NIR are longer than in the VIS range). Furthermore, the observation geometry must allow for a dark NIR background, or at least reasonable contrast between rainbow and background.

Figure 2 depicts an example where a camera on a tripod was used to record both VIS and NIR images of a natural rainbow. The NIR filter used to record this image had a cut-on wavelength of $700 \mathrm{~nm}$. This figure demonstrates quite nicely the angular shift of the NIR rainbow relative to the VIS rainbow. Note, for example, how the NIR rainbow is shifted slightly further from the tree tops at the bottom left, relative to the position of the VIS rainbow. Because red is the outer-most color in the primary VIS rainbow and the inner-most color in the secondary VIS rainbow, the primary NIR rainbow is just outside the VIS primary and the NIR secondary is inside the VIS secondary.
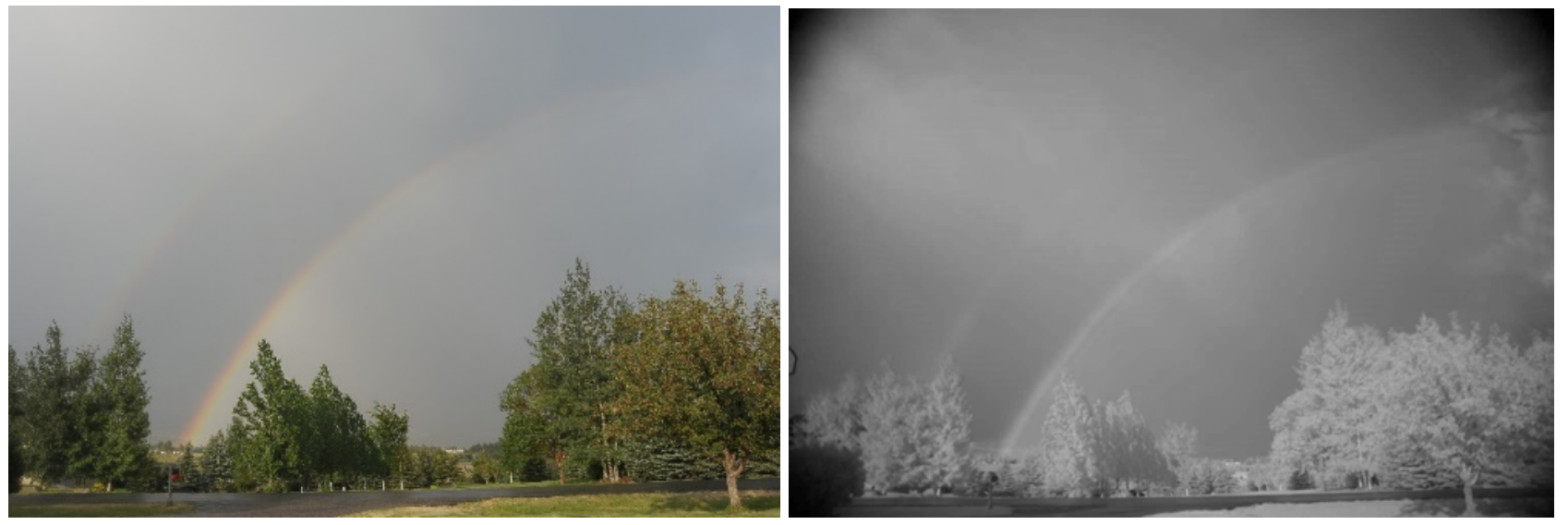

Figure 2. Rainbow photographed in the VIS (left) and NIR (right) from the same position with the same camera in Bozeman, MT.

\subsection{Glories}

The glory is a small set of circular rings that often can be observed from airplanes around the airplane shadow or antisolar point. ${ }^{10}$ The glory is almost an everyday sight during flights over clouds. A convenient method to locate the antisolar direction is to look for the end (or beginning) of the contrail shadow. Figure 3a depicts a visible photo recorded during a flight from Germany to the U.S. The glory is centered around the end of the contrail shadow (where the airplane shadow would be if the observer were closer to the cloud) and shows a series of at least three colored rings. The corresponding original NIR photo (Fig. 3b) also shows the NIR glory with up to three bright rings. The variations between maximum and minimum brightness amount just to a few percent, but the rings are still easily detectable. The most pronounced change of glories in the NIR compared to the VIS is the angular size of the glory rings, which increase with wavelength. In Fig. 3, the angular size of the outer edge of the first NIR glory ring increased by about $30 \%$ compared to the outer red edge in the VIS image.

\subsection{Halos}

In the late 20th century, many theoretical researchers studied cirrus clouds, which are the typical sources of halos in the earth's atmosphere. A typical problem addressed was the NIR transmission of radiation through such clouds. Although several typical halo features were predicted at NIR wavelengths, it was not until recently, that the first NIR observation of a $22^{\circ}$ halo has been reported. ${ }^{5}$

Figure 4 depicts a VIS and a NIR photo of a $22^{\circ}$ halo, with the sun being blocked by a pole, observed in Bozeman, MT on 20 June 2016. The sky started to be covered with thin layers of cirrus clouds in the late morning, and initially it was 
possible to observe a circum-horizontal arc (a brightly colored halo ring around part of the horizon). Unfortunately, this was already gone when the NIR camera was set up, but $22^{\circ}$ halo rings still could be observed for a couple of minutes.
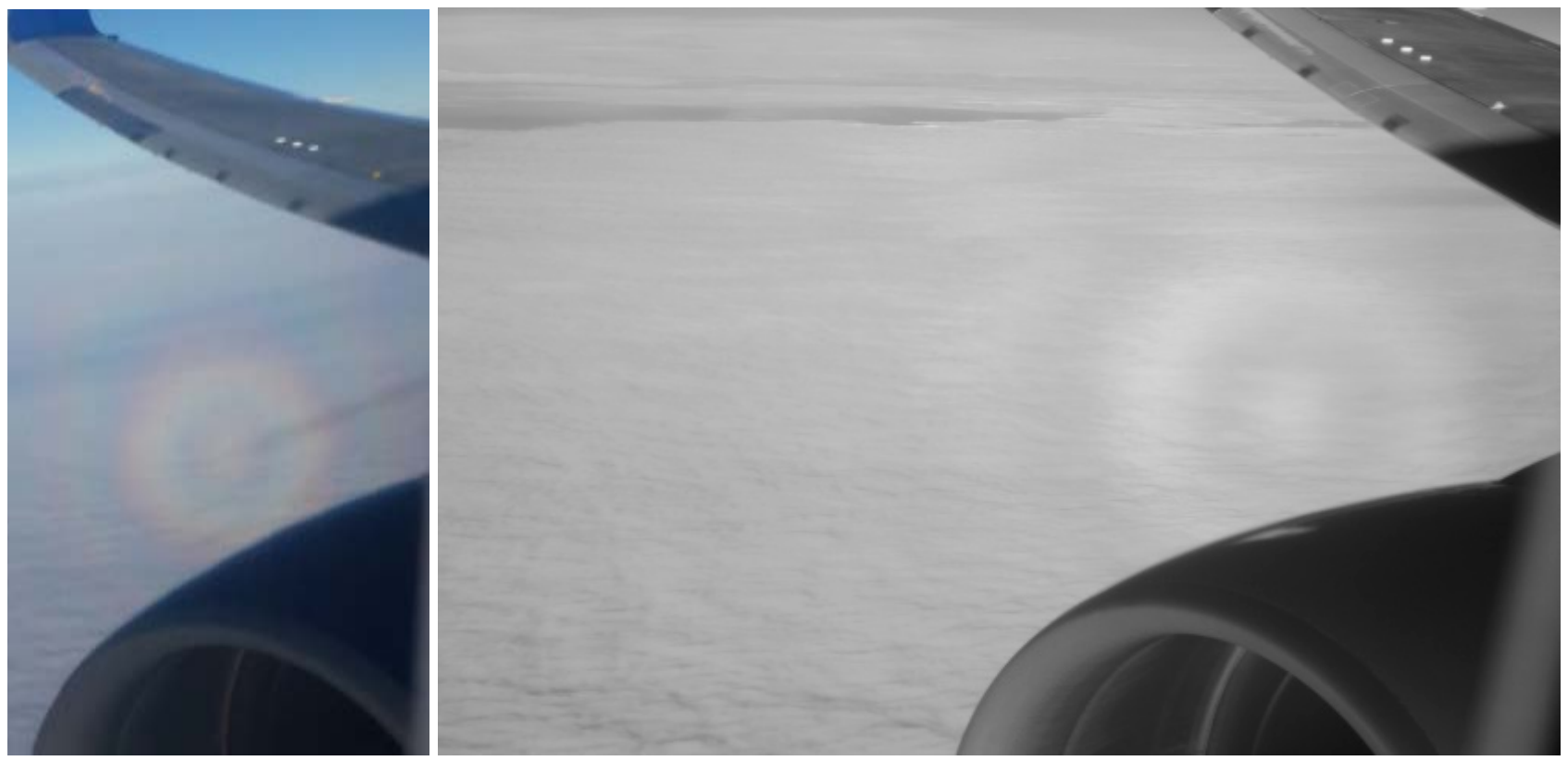

Figure 3. Glory observed from a flight on 25 September 2016 fom Frankfurt to Las Vegas: (left) smartphone VIS image; (right) NIR image.

One can also see some small clouds inside the halo, which can be seen with even better contrast in the NIR owing to reduced Rayleigh scattering in the clear sky at NIR wavelengths, which leads to improved contrast between sky and cloud (a more pronounced example of the same phenomenon that has long led black-and-white photographers to photograph scenery with a red filter). The handheld VIS camera was slightly displaced from the position of the tripodmounted NIR camera, but both used the same focal length, so they have essentially identical fields of view.

The very small dispersion of ice means that the shift of the inner ring edges between red light at $650 \mathrm{~nm}$ and NIR radiation at $850 \mathrm{~nm}$ is only about $0.3^{\circ}$, which is very difficult to see. Figure $4 \mathrm{~b}$ is only slightly modified with respect to the original NIR image. It was first converted to a grey-scale image and then an auto contrast was applied (changes made using Adobe Photoshop).

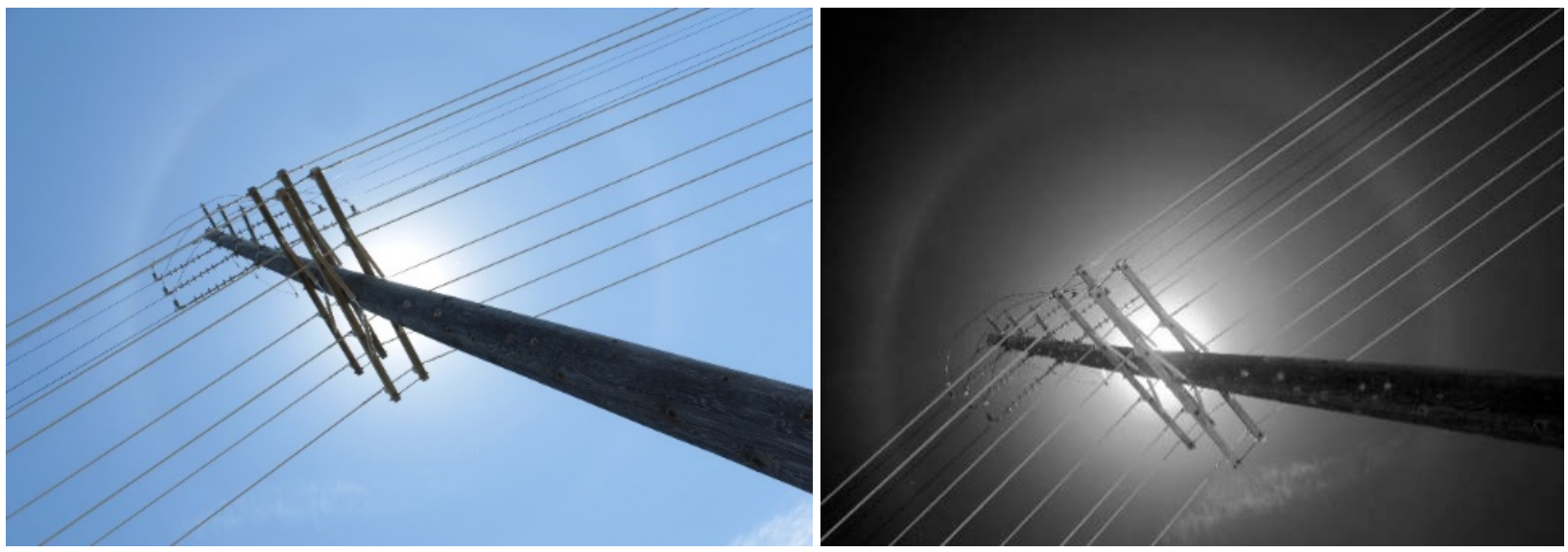

Figure 4. (left) VIS and (right) NIR photos of a $22^{\circ}$ halo from nearly the same position, observed on 20 June 2016 in Bozeman, MT. 


\subsection{Mirages}

As mentioned above, observation problems for mirages could only occur for narrow-band cameras near wavelengths with strong molecular absorption. This is not a problem with the broadband NIR filter used on our camera, so mirage detection should not be affected significantly by gaseous absorption in the atmosphere. Here we focus on one specific common example of inferior mirages seen in imagers of cars on a street in full sunshine, which heats the pavement and the adjacent air. The cars themselves, as well as other objects, such as buildings and traffic lights, typically scatter incident solar radiation without significantly favoring the NIR, but vegetation appears much brighter in the NIR. If headlights of cars are turned on, we expect no pronounced difference between NIR and VIS wavelengths if incandescent light sources are used. However, if the headlights use light emitting diodes, the NIR image may not show them at all.

Figure 5 shows typical VIS and NIR images recorded one after the other (slightly different scenes) with a 600-mm lens in Bozeman, MT on 19 June 2016. The NIR camera was mounted on a tripod, whereas the VIS camera was handheld close to the same position. The traffic lights are at street intersections at distances of approximately $800 \mathrm{~m}$ and $400 \mathrm{~m}$.

The distant cars in the VIS image show multiple inferior mirages, seen best for the strongly scattered light from the white car paint. The NIR image, on the other hand, shows the best mirages for the halogen headlights, which emit a lot of NIR radiation. These would not be visible if visible LED headlights were used, as can be guessed from the red or green LED traffic lights, which are not seen in the NIR. One can notice at least a four-fold inferior mirage.
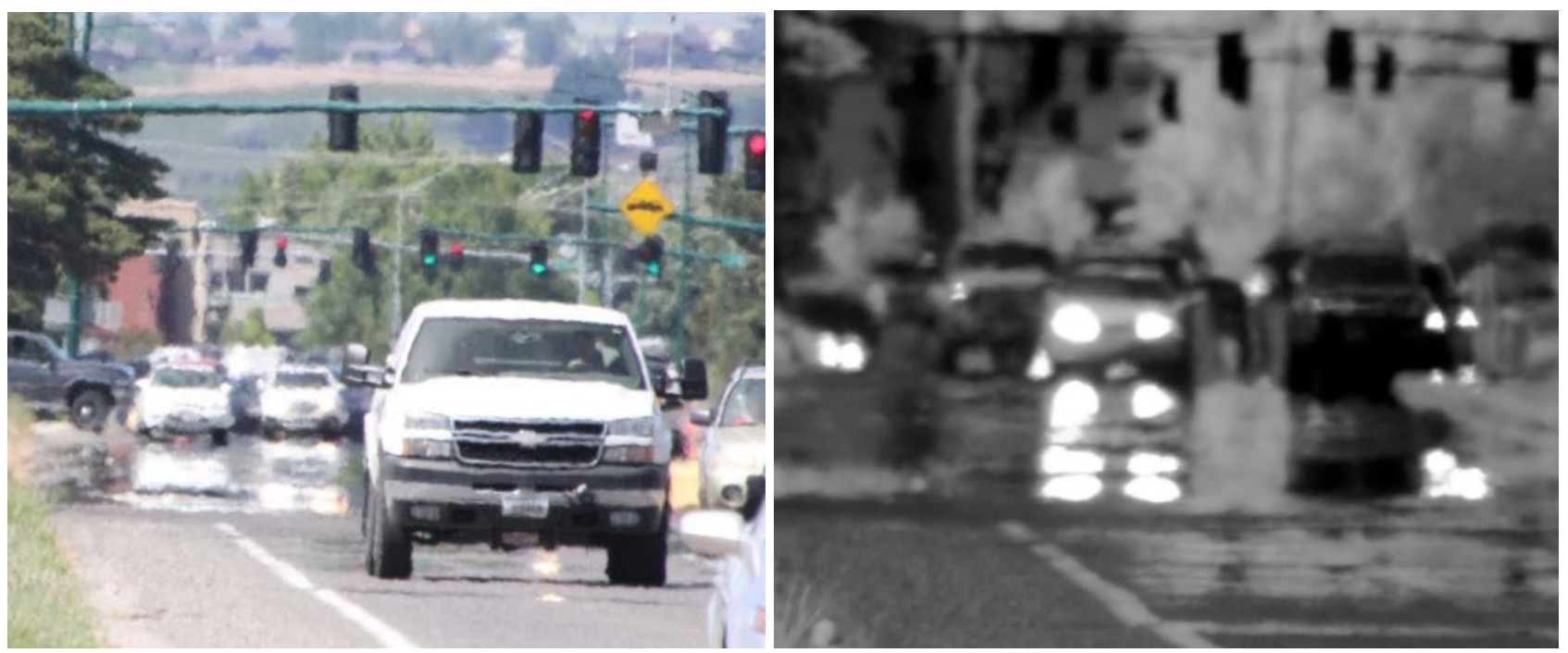

Figure 5. Observations of multiple inferior mirages on S. 19th Ave. in Bozeman, MT on 19 June 2016: (left) VIS mirage; (right) example of expanded NIR mirage with at least 4 mirage head lights, transferred to gray scale and contrast enhanced.

\section{CONCLUSION}

There are no fundamental reasons that atmospheric optical phenomena should not be observable in the NIR spectral range of approximately $780-1100 \mathrm{~nm}$. Indeed, they can be observed using a standard digital single-lens-reflex (DSLR) camera that has been modified to block visible light and record only NIR radiation. We have presented NIR photographs of naturally occurring rainbows, glories, halos, and mirages. These images offer a new way of teaching the underlying physics principles as a function of wavelength.

\section{Acknowledgment}

JAS acknowledges support from the U.S. Air Force Office of Scientific Research under agreement number FA955014-10140. This paper summarizes material published previously in [5]. 


\section{REFERENCES}

[1] Greenler, R. and Lynch, D. K., "Light and Color in Nature: A return to Optics' roots," Opt. Photonics News 30-37 (2011); see also Appl. Opt. 33(21) (1994), 37(9) (1998), 42(3) (2003), 44(27) (2005), 47(34) (2008), 50(28) (2011), 54(4) (2015), 56(19) (2017).

[2] R. Greenler, Rainbows, Halos and Glories, Cambridge University Press (1980).

[3] Lynch, D. K. and Livingston, W., [Color and Light in Nature, 2 ${ }^{\text {nd }}$. Ed.], Cambridge Univ. Press (2001).

[4] Mangold, K., Shaw, J. A., and Vollmer, M., “The Physics of Near-Infrared Photography,” Eur. J. Phys. 34(6), S5171 (2013).

[5] Vollmer, M. and Shaw, J. A., “Atmospheric Optics in the Near Infrared,” Appl. Opt. 56(19), G145-155 (2017).

[6] Vollmer, M., Möllmann, K.-P., and Shaw, J. A., "The optics and physics of near infrared imaging," Proc. SPIE 9793, $97930 Z$ (2015).

[7] Sandidge, D., [Digital Infrared Photography], Wiley, New York (2009).

[8] Farace, J., [Complete Guide to Digital Infrared Photography], Lark Books, Asheville, NC (2006).

[9] Greenler, R., "Infrared Rainbow," Science, 173(4003), 1231-1232 (1971).

[10] Shaw, J. A., [Optics in the Air: Observing optical phenomena through airplane windows], SPIE Press, Bellingham, WA (2017). 